\title{
On the Convergence of Collocation Methods for Symm's Integral Equation on Open Curves
}

\author{
By M. Costabel, V. J. Ervin, and E. P. Stephan*
}

\begin{abstract}
Recently, Costabel and Stephan in [8] presented convergence proofs for collocation with piecewise linear trial functions for Symm's integral equation on plane closed curves with corners. In this article we prove the convergence of the above collocation method in the case of open curves. We derive asymptotic error estimates in Sobolev norms and analyze the effect of graded meshes. Numerical experiments based on the implementation of [6] show experimental orders of convergence which confirm our theoretical results on the asymptotic rates of convergence.
\end{abstract}

0. Introduction. In this paper we give convergence proofs and asymptotic error estimates in Sobolev norms for collocation with piecewise linear spline trial functions applied to the integral equation of the first kind with single layer potential ("Symm's integral equation") on open curves. Our results are obtained by a refinement of the analysis in [8] where the collocation method for closed, polygonal curves is investigated. Besides [8], convergence proofs and error estimates for boundary element collocation methods have been only available in the following cases: Fredholm integral equations of the second kind [4], [5], one-dimensional pseudodifferential equations and singular integral equations with piecewise smooth coefficients on smooth closed curves [2], [3], [12], [17], [18], [19], [20]. In this article we use again an idea of Arnold and Wendland [2], namely considering Dirac delta functions (the "test functions" in the collocation method) as second derivatives of piecewise linear functions. Therefore, similar results as presented here should be possible for splines of higher odd order. Corresponding results for even-order splines are not yet available; compare however [10]. The method of Fourier series that yields the convergence proof in the case of a smooth, closed boundary [18] cannot be applied to open curves where the endpoints can be viewed as vertices with angle $2 \pi$.

For brevity we consider only the case where $\Gamma$ is a smooth arc with endpoints $z_{1}$ and $z_{2}$. All the results remain valid in the case of $\Gamma$ consisting of finitely many open arcs which do not intersect themselves. Furthermore, let $\tilde{\Gamma}$ be a smooth, closed curve containing $\Gamma$. Then the Sobolev spaces $H^{s}(\tilde{\Gamma})$ are defined for $s>0$ as being the restrictions of $H^{s+1 / 2}\left(\mathbf{R}^{2}\right)$ to $\tilde{\Gamma}$ and for $s<0$ by duality: $H^{s}(\tilde{\Gamma})=\left(H^{-s}(\tilde{\Gamma})\right)^{\prime}$ and $H^{0}(\tilde{\Gamma})=L^{2}(\tilde{\Gamma})$. These spaces (see [14]) are used to define the corresponding spaces of distributions on $\Gamma$, namely, for any real $s$,

$$
\tilde{H}^{s}(\Gamma)=\left\{u \in H^{s}(\tilde{\Gamma}): \operatorname{supp} u \subset \bar{\Gamma}\right\}
$$

Received June 16, 1987; revised September 21, 1987.

1980 Mathematics Subject Classification (1985 Revision). Primary 65N35, 65R20; Secondary 65D07, 45L10.

${ }^{*}$ The third author was supported by NSF grants DMS-8501797 and DMS-8603954. 
and

$$
H^{s}(\Gamma)=\left\{\left.u\right|_{\Gamma}: u \in H^{s}(\tilde{\Gamma})\right\} .
$$

The paper is organized as follows: In Section 1 we present from [8] some facts on the convergence of general projection methods (Lemma 1.2). They are stated in a form which is convenient for the application to collocation methods and allows easy incorporation of localization arguments. For the localization principle, see [12], [15]. Utilizing those facts, we prove convergence, stability and error estimates for the collocation method with piecewise linear splines applied to Symm's integral equation (Theorem 1.1).

In Section 2 we investigate the asymptotic order of convergence in weighted Sobolev norms. We prove a new approximation result in those weighted norms (Lemma 2.1) and we show that (analogously to the case of a polygonal curve $\Gamma$ ) the use of suitably graded meshes improves convergence (Theorem 2.2).

In Section 3 we present numerical results for the implementation of our collocation scheme applied to two sample problems: (i) the lateral crack and (ii) the interior crack of a membrane. In both examples the computed experimental order of convergence confirms our theoretical error analysis of Section 2.

1. Collocation for the Single Layer Potential. The integral equation of the first kind with logarithmic kernel ("Symm's integral equation") is one of the classical integral equations of potential theory. It arises, e.g., from a single layer representation for the solution of the Dirichlet problem, and it has also well-known applications in conformal mappings. For the integral equation of the first kind on an open piece of a curve $\Gamma$,

$$
V u=g
$$

we consider convergence of the collocation method with piecewise linear trial functions. Here the operator $V$ of the single layer potential is defined by

$$
V u(z):=-\frac{1}{\pi} \int_{\Gamma} u(\varsigma) \log |z-\varsigma| d s(\varsigma),
$$

where $s(\zeta)$ is the arc length on $\Gamma$. It is known [7] that $V: \tilde{H}^{s}(\Gamma) \rightarrow H^{s+1}(\Gamma)$ is continuous and bijective for all $s \in(-1,0)$, provided the analytic capacity of $\Gamma$ is not equal to one. We shall assume this in the sequel.

For the collocation method we need a grid $\tilde{\Delta}=\left\{x_{1}, \ldots, x_{N}\right\} \subset \bar{\Gamma}$, the $x_{j}$ being both the collocation points and the mesh points of the trial functions. By $S^{1}(\tilde{\Delta})$ we denote the $N$-dimensional space of splines of order 1 , i.e., each $u \in S^{1}(\tilde{\Delta})$ is a continuous function on $\bar{\Gamma}$ that is a linear function of the arc length on each segment $x_{j} x_{j+1}, j=0, \ldots, N-1$. Let $h:=\max \left\{\left|x_{j+1}-x_{j}\right|: j=0, \ldots, N-1\right\}$. We do not impose a uniformity condition on $\tilde{\Delta}$, but assume only that $h \rightarrow 0$ as $N$ tends to infinity.

We require that the endpoints $z_{1}, z_{2}$ are grid points, namely $z_{1}=x_{0}, z_{2}=x_{N}$ and define

$$
\begin{gathered}
\tilde{S}^{1}(\tilde{\Delta}):=\left\{v \in S^{1}(\tilde{\Delta}) \mid v\left(z_{j}\right)=0, j=1,2\right\} \\
S_{\rho}^{1}(\tilde{\Delta}):=\left\{\frac{1}{\rho} v \mid v \in S^{1}(\tilde{\Delta})\right\} .
\end{gathered}
$$


Here the weight function $\rho \geq 0$ satisfies $\rho \in C^{\infty}\left(\mathbf{R}^{2} \backslash\left\{z_{1}, z_{2}\right\}\right)$ and

$$
\rho(z)=\left|z-z_{j}\right| \text { in a neighborhood of } z_{j}, j=1,2 \text {. }
$$

Thus $\tilde{S}^{1}(\tilde{\Delta})$ and $S_{\rho}^{1}(\tilde{\Delta})$ have dimension $N-2$, and the elements of $S_{\rho}^{1}(\tilde{\Delta})$ are continuous on $\Gamma$ and (for $h$ small enough) take constant values on the two grid segments that end at the crack tips $z_{j}$. The $N-2$ collocation equations are

$$
V u_{N}\left(x_{n}\right)=g\left(x_{n}\right), \quad x_{n} \in \tilde{\Delta} \backslash\left\{z_{1}, z_{2}\right\}, \text { with } u_{N} \in S_{\rho}^{1}(\tilde{\Delta}) .
$$

In order to show convergence of the collocation method (1.3), we need the weighted Sobolev space

$$
\tilde{H}_{\rho}^{1 / 2}(\Gamma):=\frac{1}{\rho} \tilde{H}^{1 / 2}(\Gamma)=\left\{u \mid \rho u \in \tilde{H}^{1 / 2}(\Gamma)\right\}
$$

with the norm

$$
\|u\|_{\tilde{H}_{\rho}^{1 / 2}}:=\|\rho u\|_{\tilde{H}^{1 / 2}} .
$$

In the following we prove the following convergence and stability result for the collocation scheme (1.3).

THEOREM 1.1. There exist $N_{0} \in \mathrm{N}$ and $C \geq 0$ such that for all $g \in H^{1 / 2}(\Gamma)$ with $u=V^{-1} g \in \tilde{H}^{-1 / 2}(\Gamma)$ satisfying $\rho u \in \tilde{H}^{1 / 2}(\Gamma)$ there exists a unique solution $u_{N} \in S_{\rho}^{1}(\tilde{\Delta})$ of $(1.3)$, and

$$
\left\|u_{N}\right\|_{\tilde{H}_{\rho}^{1 / 2}(\Gamma)} \leq C\left(\|u\|_{\tilde{H}_{\rho}^{1 / 2}(\Gamma)}+\|u\|_{H^{-1 / 2}(\Gamma)}\right) \quad(\text { stability })
$$

and

$$
\begin{aligned}
&\left\|u-u_{N}\right\|_{\tilde{H}_{\rho}^{1 / 2}(\Gamma)} \leq C \inf \left\{\|u-\tilde{u}\|_{H^{-1 / 2}(\Gamma)}+\|u-\tilde{u}\|_{\tilde{H}_{\rho}^{1 / 2}(\Gamma)}\right.\left.\mid \tilde{u} \in S_{\rho}^{1}(\tilde{\Delta})\right\} \\
& \text { (quasioptimality). }
\end{aligned}
$$

For the proof of Theorem 1.1 we follow the ideas in [8] and therefore need some results on the convergence of projection methods including compact perturbations and spaces with two norms. Such results are well known [11], [13], [16], but we present from [8] a formulation which is particularly adapted to the present case.

Let $X$ and $Y$ be Banach spaces and $A: X \rightarrow Y$ be bijective and continuous. For the approximate solution of the equation

$$
A u=f
$$

we assume that we have a sequence of finite-dimensional subspaces

$$
V_{N} \subset X, \quad T_{N} \subset Y^{\prime}, \quad \operatorname{dim} V_{N}=\operatorname{dim} T_{N}^{\prime}<\infty,
$$

and we replace Eq. (1.6) by the relation for $u_{N} \in V_{N}$

$$
\left\langle t, A u_{N}\right\rangle=\langle t, f\rangle \quad \text { for all } t \in T_{N}
$$

where $\langle$,$\rangle denotes the duality between Y$ and its dual $Y^{\prime}$. We make the following assumptions:

(i) There exist bounded operators $P_{N}: Y^{\prime} \rightarrow T_{N}$ that converge on $Y^{\prime}$ strongly to the identity operator.

(ii) There is a Banach space $X_{0}$, continuously embedded in $X$ (hence, $\|x\|_{X} \leq$ $C\|x\|_{X_{0}}$ for all $x \in X_{0}$ and some constant $C$ ). 
(iii) For all $N$ there holds $V_{N} \subset X_{0}$. that

(iv) For all $N$ we are given a mapping $Q_{N}: V_{N} \rightarrow T_{N}$ and a constant $M$ such

$$
\left|\left\langle Q_{N} u, A w\right\rangle\right| \leq M\|u\|_{X}\|w\|_{X_{0}} \quad \text { for all } v \in V_{N}, w \in X_{0}, N \in \mathbf{N} .
$$

(v) There exists a collectively compact sequence of operators $C_{N}: X \rightarrow X^{\prime}$ in the sense of [1] and a constant $\gamma>0$ such that

$$
\left|\left\langle Q_{N} v, A v\right\rangle+\left\langle C_{N} v, v\right\rangle\right| \geq \gamma\|v\|_{X}^{2} \quad \text { for all } v \in V_{N}, N \in \mathbf{N}
$$

LEMMA 1.2 ([8], [9]). Under the above conditions (i) $-(\mathrm{v})$ there exists $N_{0} \in \mathbf{N}$ such that for all $N \geq N_{0}$ the system (1.7) has a unique solution $u_{N} \in V_{N}$ for any $f \in Y$. There is a constant $C$ such that for this approximate solution $u_{N}$ and the true solution $u$ there holds

$$
\begin{gathered}
\left\|u_{N}\right\|_{X} \leq C\|u\|_{X_{0}} \quad \text { for all } u \in X_{0}, \quad N \geq N_{0}, \\
\left\|u-u_{N}\right\|_{X} \leq C \inf \left\{\|u-\tilde{u}\|_{X_{0}} \mid \tilde{u} \in V_{N}\right\} .
\end{gathered}
$$

Remark. We shall need the lemma only for the case of $Q_{N}$ and $C_{N}$ not depending on $N$. Thus $Q_{N} \equiv Q: X_{0} \rightarrow Y^{\prime}$ will be a linear operator satisfying

$$
Q V_{N} \subset T_{N} \text { for all } N \in \mathrm{N}
$$

and $C_{N} \equiv C: X \rightarrow X^{\prime}$ will be a compact operator, or equivalently, the quadratic form $v \mapsto\langle C v, v\rangle$ appearing in (1.9) will be completely continuous on $X$.

Proof of Theorem 1.1. We set $X:=\tilde{H}_{\rho}^{1 / 2}$ and $X_{0}:=\tilde{H}_{\rho}^{1 / 2} \cap \tilde{H}^{-1 / 2}(\Gamma)$ with the norm

$$
\|u\|_{X_{0}}^{2}:=\|u\|_{\tilde{H}_{\rho}^{1 / 2}}^{2}+\|u\|_{\tilde{H}^{-1 / 2}(\Gamma)}^{2} .
$$

With $A:=V$ and $Y:=A X$, the hypotheses of Lemma 1.2 are satisfied, as we will show in the following.

1. First we observe that $V$ is well defined on $X$, since any $u \in \tilde{H}_{\rho}^{1 / 2}$ belongs to $\tilde{H}^{-1 / 2-\varepsilon}(\Gamma), \varepsilon>0$, and $V$ is defined on $\tilde{H}^{-1 / 2-\varepsilon}(\Gamma)$ by continuous extension from $\tilde{H}^{-1 / 2}(\Gamma) . V$ is also injective in $X$ since $V: \tilde{H}^{-1 / 2+\sigma}(\Gamma) \rightarrow H^{1 / 2+\sigma}(\Gamma),|\sigma|<1 / 2$, is bijective. The latter result was shown in [7].

2. Next we define

$$
\stackrel{\circ}{S}^{-1}(\tilde{\Delta}):=\left\{\phi \in S^{-1}(\tilde{\Delta}) \mid \operatorname{supp} \phi \cap\left\{z_{1}, z_{2}\right\}=\varnothing\right\}
$$

where

$$
S^{-1}(\tilde{\Delta}):=\operatorname{span}\left\{\delta\left(x-x_{n}\right) \mid n=1, \ldots, N\right\}
$$

Then

$$
\operatorname{dim} S_{\rho}^{1}(\tilde{\Delta})=\operatorname{dim} \stackrel{\circ}{S}^{-1}(\tilde{\Delta})=N-2
$$

and $u \in S_{\rho}^{1}(\tilde{\Delta})$ implies $\rho D^{2} \rho u \in \stackrel{\circ}{S}^{-1}(\tilde{\Delta})$. Thus we set $V_{N}:=S_{\rho}^{1}(\tilde{\Delta}), T_{N}:=$ $\stackrel{\circ}{S}^{-1}(\tilde{\Delta})$ and $Q:=\rho D^{2} \rho$, where $D^{2}$ denotes the second distributional derivative with respect to arc length. Then the collocation equations (1.3), or (1.7), are given by

$$
\left\langle\rho D^{2} \rho v, V u\right\rangle=\left\langle\rho D^{2} \rho v, g\right\rangle \quad \text { for all } v \in S_{\rho}^{1}(\tilde{\Delta})\left(\text { with } t=\rho D^{2} \rho v\right) \text {. }
$$


3. Now we check the hypotheses of the lemma: Assumption (ii) is trivial. Concerning assumption (iii), we note that every $v \in S_{\rho}^{1}(\tilde{\Delta})$ is continuous on $\Gamma$. Thus, $V_{N} \subset X_{0}$ holds. It remains to show the estimates (1.8) and (1.9).

We remark that outside the neighborhoods of the endpoints of $\Gamma$, the spaces $X$ and $X_{0}$ coincide with $H^{1 / 2}(\tilde{\Gamma})$, where $\tilde{\Gamma}$ is a smooth curve. For the latter case, the estimates (1.8), (1.9) are well known from the calculus of pseudodifferential operators, namely there exists a $\gamma>0$ such that for all $v \in H^{1 / 2}(\tilde{\Gamma})$

$$
\left\langle D^{2} v, V v\right\rangle \geq \gamma\|v\|_{H^{1 / 2}(\tilde{\Gamma})}^{2}
$$

Therefore, by the localization principle (see Lemma 1.4 in [8]), it suffices to show the estimates (1.8) and (1.9) for $\Gamma=\mathbf{R}_{+}=[0, \infty)$.

Thus, we only consider $\Gamma=\mathbf{R}_{+}$and the bilinear form

$$
b(v, w):=\langle D \rho v, D \rho V w\rangle_{L^{2}\left(\mathbf{R}_{+}\right)},
$$

where $v, w \in C_{0}^{\infty}\left(\mathbf{R}_{+}\right)$with support in a fixed compact set and $\rho(z)=|z|$ on the support of $v$ and $w$. Thus, $(\rho v)(x)=x v(x)$ on $\mathbf{R}_{+}$. For all $v, w \in C_{0}^{\infty}\left(\mathbf{R}_{+}\right)$we have

$$
b(v, w)=b_{1}(v, w)+b_{2}(v, w)
$$

with

$$
b_{1}(v, w):=\langle D \rho v, V D \rho w\rangle, \quad b_{2}(v, w):=\langle D \rho v, V w\rangle,
$$

since $\rho D V=V D \rho$ on $\mathbf{R}_{+}$. Furthermore, the following estimates hold with $\gamma>0$ and $M$ independent of $v, w$ :

$$
\begin{aligned}
& \left|b_{1}(v, w)\right| \leq M\|v\|_{\tilde{H}_{\rho}^{1 / 2}(\Gamma)}\|w\|_{\tilde{H}_{\rho}^{1 / 2}(\Gamma)} \\
& \operatorname{Re}\left(b_{1}(v, v)+\langle C v, v\rangle\right) \geq \gamma\|v\|_{\tilde{H}_{\rho}^{1 / 2}(\Gamma)}^{2}
\end{aligned}
$$

with a compact operator $C$,

$$
\left|b_{2}(v, w)\right| \leq M\|v\|_{\tilde{H}_{\rho}^{1 / 2}(\Gamma)}\left(\|w\|_{\tilde{H}_{\rho}^{1 / 2}(\Gamma)}+\|w\|_{\tilde{H}^{-1 / 2}(\Gamma)}\right) .
$$

These estimates follow from the following known properties of the operator $V$ (see [8]):

$$
\begin{gathered}
|\langle D \rho v, V D \rho w\rangle| \leq M\|D \rho v\|_{\tilde{H}^{-1 / 2}(\Gamma)}\|D \rho w\|_{\tilde{H}^{-1 / 2}(\Gamma)} \\
\operatorname{Re}\langle D \rho v, V D \rho v\rangle \geq \gamma\|D \rho v\|_{\tilde{H}^{-1 / 2}(\Gamma)}^{2} \\
\mid\langle D \rho v, V w\rangle \leq M\|D \rho v\|_{\tilde{H}^{-1 / 2}(\Gamma)}\|w\|_{\tilde{H}^{-1 / 2}(\Gamma)}
\end{gathered}
$$

Here we remark that the norms

$$
\|D \rho v\|_{\tilde{H}^{-1 / 2}(\Gamma)} \quad \text { and } \quad\|\rho v\|_{\tilde{H}^{1 / 2}(\Gamma)}\left(=:\|v\|_{\tilde{H}_{\rho}^{1 / 2}(\Gamma)}\right)
$$

are equivalent, since the differential operator $D$ is an isomorphism from $\tilde{H}^{1 / 2}(\Gamma)$ onto a closed subspace of $\tilde{H}^{-1 / 2}(\Gamma)$.

Altogether, with (1.12)-(1.15), there follows that for the bilinear form $b(\cdot, \cdot)$ in (1.11) we can apply Lemma 1.2, which yields Theorem 1.1 . 
2. Order of Convergence. From the error estimates in Theorem 1:1 one can derive convergence orders simply by using results on the order of best spline approximation in the respective norms. We need here a result on spline approximation in weighted Sobolev spaces similar to the result derived in [8], [9], where polygonal domains with angles $\omega \neq 2 \pi$ are considered. The limit case $\omega=2 \pi$ representing an open piece of a curve, considered here, is not covered by the analysis in [8], [9].

We assume that $u \in \tilde{H}^{-1 / 2}(\Gamma)$ is the solution of the equation $V u=f$ with $f$ sufficiently smooth, where $\Gamma$ is an open piece of a curve. Then $u$ has the following properties:

(i) $u \in H^{-\varepsilon}(\Gamma), \rho u \in H^{1-\varepsilon}$ (any $\varepsilon>0$ ),

(ii) $\rho u=O\left(\rho^{1 / 2}\right), D \rho u=O\left(\rho^{-1 / 2}\right)$ near the endpoints of $\Gamma$.

We approximate $u$ by its interpolation $\tilde{u}$ in $S_{\rho}^{1}(\Delta)$. Thus, if we define

$$
w:=\rho u, \quad \tilde{w}:=\rho \tilde{u},
$$

then $\tilde{w}$ is the piecewise linear interpolant of $w$ with nodes in $\tilde{\Delta}$. Note that $w$ is continuous on $\Gamma$ and vanishes at the endpoints of $\Gamma$. Now for the error estimate it suffices to consider a neighborhood of the endpoints, because outside such a neighborhood, $u$ is smooth and therefore one has an approximation of order $h^{3 / 2}$ in the $H^{1 / 2}$-norm.

We consider a one-sided neighborhood of one endpoint and assume that it is parametrized by the unit interval. We further assume that

$$
\rho(x)=x
$$

and $u$ is given on $[0,1]$ with the properties (i) and (ii) above. We define $\tilde{u}, w$ and $\tilde{w}$ as above. We assume that the grid $\tilde{\Delta}$ has the form

$$
x_{j}=(j h)^{\beta}, \quad j=0, \ldots, N=1 / h,
$$

with some $\beta \geq 1$. We have to estimate the two norms on $[0,1]$,

$$
\|u-\tilde{u}\|_{\tilde{H}^{-1 / 2}} \text { and }\|w-\tilde{w}\|_{\tilde{H}^{1 / 2}} .
$$

LEMMA 2.1. There is a constant $C$ such that

$$
\|u-\tilde{u}\|_{\tilde{H}^{-1 / 2}}+\|w-\tilde{w}\|_{\tilde{H}^{1 / 2}} \leq\left\{\begin{array}{ll}
C h & \text { for } \beta>2, \\
C h^{(\beta / 2)-\varepsilon} & \text { for } 1 \leq \beta<2(\varepsilon>0) .
\end{array} .\right.
$$

Proof. From the estimates (ii) above we find for $x \in\left[x_{j}, x_{j+1}\right], 1 \leq j \leq N-1$ :

$$
\begin{aligned}
|(w-\tilde{w})(x)| & \leq \frac{1}{8}\left|x_{j+1}-x_{j}\right|^{2} \sup _{\xi \in\left[x_{j}, x_{j+1}\right]}\left|w^{\prime \prime}(\xi)\right| \\
& \leq C h^{\beta / 2}(j+1)^{\beta / 2-2}
\end{aligned}
$$

and

$$
\begin{aligned}
\left|(w-\tilde{w})^{\prime}(x)\right| & \leq\left|x_{j+1}-x_{j}\right| \sup _{\xi \in\left[x_{j}, x_{j+1}\right]}\left|w^{\prime \prime}(\xi)\right| \\
& \leq C h^{-\beta / 2}(j+1)^{-\beta / 2-1} .
\end{aligned}
$$

We then estimate the following integrals for $1 \leq j \leq N-1$ :

$$
\begin{gathered}
\mathscr{J}_{j}{ }^{1}:=\int_{x_{j}}^{x_{j+1}}|(w-\tilde{w})(x)|^{2} d x \leq C h^{2 \beta}(j+1)^{2 \beta-5}, \\
\mathscr{J}_{j}^{2}:=\int_{x_{j}}^{x_{j+1}}\left|(w-\tilde{w})^{\prime}(x)\right|^{2} d x \leq C(j+1)^{-3} .
\end{gathered}
$$


This gives on $\left[x_{1}, 1\right]$

$$
\|w-\tilde{w}\|_{L^{2}\left[x_{1}, 1\right]}^{2}=\sum_{j=1}^{N-1} \mathscr{J}_{j}^{1} \leq C h^{2 \beta} \sum_{j=2}^{N} j^{2 \beta-5},
$$

where the sum on the right-hand side is bounded for $2 \beta-5<-1$ and bounded by $C N^{2 \beta-4}=C h^{4-2 \beta}$ for $2 \beta-5>-1$. Therefore, we obtain

$$
\|w-\tilde{w}\|_{L^{2}\left[x_{1}, 1\right]} \leq\left\{\begin{array}{l}
C h^{2} \\
C h^{\beta}
\end{array} \text { for } \beta\left\{\begin{array}{l}
> \\
<
\end{array}\right\} \begin{array}{l}
2 \\
2
\end{array} .\right.
$$

Furthermore, using the estimate for $\mathscr{J}_{j}^{2}$, we find

$$
\left\|(w-\tilde{w})^{\prime}\right\|_{L^{2}\left[x_{1}, 1\right]}^{2} \leq C h^{0} \quad \text { for all } \beta .
$$

Interpolation between (2.2) and (2.3) gives

$$
\|w-\tilde{w}\|_{H^{1 / 2}\left[x_{1}, 1\right]} \leq C\left\{\begin{array}{l}
h \\
h^{\beta / 2}
\end{array} \text { for } \beta\left\{\begin{array}{l}
> \\
<
\end{array}\right\} \begin{array}{l}
2 \\
2
\end{array} .\right.
$$

In order to get corresponding norm estimates on $\left[0, x_{1}\right]$, we set

$$
w=w_{0}+w_{1} \quad \text { with } w_{0}=\left\{\begin{array}{ll}
\tilde{w} & \text { on }\left[0, x_{1}\right], \\
w & \text { on }\left[x_{1}, 1\right],
\end{array} \quad w_{1}= \begin{cases}w-\tilde{w} & \text { on }\left[0, x_{1}\right] \\
0 & \text { on }\left[x_{1}, 1\right]\end{cases}\right.
$$

where

$$
\tilde{w}(x)=\frac{w\left(x_{1}\right)}{x_{1}} x \quad \text { on }\left[0, x_{1}\right]
$$

coincides with $w$ at $x=0$ and at $x=x_{1}$. Hence,

$$
w_{0}-\tilde{w}= \begin{cases}0 & \text { on }\left[0, x_{1}\right] \\ w-\tilde{w} & \text { on }\left[x_{1}, 1\right]\end{cases}
$$

and therefore interpolation of the estimates (2.2) and (2.3) yields

$$
\left\|w_{0}-\tilde{w}\right\|_{\tilde{H}^{1 / 2}} \leq\left\{\begin{array}{l}
C h \\
C h^{\beta / 2}
\end{array} \text { for } \beta\left\{\begin{array}{l}
> \\
<
\end{array}\right\} \begin{array}{l}
2 \\
2
\end{array}\right.
$$

Since $w-\tilde{w}=\left(w_{0}-\tilde{w}\right)+w_{1}$, it remains to estimate $w_{1}$ on $\left[0, x_{1}\right]$. First we observe for its seminorm in $\tilde{H}^{1 / 2}$ :

$$
\begin{aligned}
\left|w_{1}\right|_{\tilde{H}^{1 / 2}}^{2}= & \iint_{|x|<2} \frac{\left|w_{1}(x)-w_{1}(y)\right|^{2}}{|x-y|^{2}} d x d y \simeq \int_{0}^{1} \frac{\left|w_{1}(x)\right|^{2}}{x} d x \\
& +\int_{0}^{1} \int_{x}^{1} \frac{\left|w_{1}(x)-w_{1}(y)\right|^{2}}{|x-y|^{2}} d y d x
\end{aligned}
$$

By the mean value theorem we have

$$
\left|w_{1}(x)\right| \leq C\left(x^{1 / 2}+x x_{1}^{-1 / 2}\right) \leq C h^{\beta / 2} \text { for } x \leq x_{1}=h^{\beta} .
$$

So for all $\beta$ and $x_{1}=h^{\beta}$ we get

$$
\int_{0}^{1} \frac{\left|w_{1}(x)\right|^{2}}{x} d x=\int_{0}^{x_{1}} \frac{\left|w_{1}(x)\right|^{2}}{x} d x \leq \int_{0}^{x_{1}} C\left(1+x h^{-\beta}\right) d x \leq C h^{\beta}
$$

and

$$
\left\|w_{1}\right\|_{L^{2}}^{2} \leq C h^{2 \beta}
$$


For the second integral on the right-hand side in (2.4) we have, after the change of variables $y=x+z$,

(2.6)

$$
\begin{aligned}
\int_{0}^{1} & \int_{0}^{1-x} \frac{\left|w_{1}(x)-w_{1}(x+z)\right|^{2}}{|z|^{2}} d x d z \\
\quad & =\int_{0}^{x_{1}} \int_{x_{1}-x}^{1-x} \frac{\left|w_{1}(x)\right|^{2}}{z^{2}} d z d x+\int_{0}^{x_{1}} \int_{0}^{x_{1}-x} \frac{\left|w_{1}(x)-w_{1}(x+z)\right|^{2}}{z^{2}} d z d x
\end{aligned}
$$

With $w_{1}\left(x_{1}\right)=0$, application of the mean value theorem gives

$$
w_{1}(x)=O\left(x^{-1 / 2}\left(x_{1}-x\right)\right)
$$

Now we can estimate

$$
\begin{aligned}
\int_{0}^{x_{1}} & \int_{x_{1}-x}^{1-x} \frac{\left|w_{1}(x)\right|^{2}}{z^{2}} d z d x \\
\quad & =\int_{0}^{x_{1} / 2} \int_{x_{1}-x}^{1-x} \frac{\left|w_{1}(x)\right|^{2}}{z^{2}} d z d x+\int_{x_{1} / 2}^{x_{1}} \int_{x_{1}-x}^{1-x} \frac{\left|w_{1}(x)\right|^{2}}{z^{2}} d z d x \leq C h^{\beta}
\end{aligned}
$$

by using the estimate (2.5) for the first integral and (2.7) for the second integral on the right-hand side.

Next we have by the mean value theorem

$$
\left|w_{1}(x)-w_{1}(x+z)\right|=z \cdot O\left(x^{-1 / 2}+h^{-\beta / 2}\right) .
$$

Furthermore, from (2.5),

$$
\left|w_{1}(x)\right|+\left|w_{1}(x+z)\right|=O\left(h^{\beta / 2}\right) .
$$

Now with (2.8) and (2.9), and $1 / 2<\varepsilon<1, x_{1}=h^{\beta}$, we can estimate the second integral on the right-hand side of $(2.6)$ as follows:

$$
\begin{aligned}
\int_{0}^{x_{1}} & \int_{0}^{x_{1}-x} \frac{\left|w_{1}(x)-w_{1}(x+z)\right|^{2}}{z^{2}} d z d x \\
& \leq C \int_{0}^{x_{1}} \int_{0}^{x_{1}-x} \frac{\left|w_{1}(x)-w_{1}(x+z)\right|^{2 \varepsilon}}{z^{2}}\left(\left|w_{1}(x)\right|+\left|w_{1}(x+z)\right|\right)^{2-2 \varepsilon} d z d x \\
& \leq C \int_{0}^{x_{1}} \int_{0}^{x_{1}-x} z^{2 \varepsilon-2}\left(x^{-1 / 2}+h^{-\beta / 2}\right)^{2 \varepsilon} h^{(1-\varepsilon) \beta} d z d x \\
& \leq C \int_{0}^{x_{1}}\left(x_{1}-x\right)^{2 \varepsilon-1}\left(x^{-\varepsilon}+h^{-\beta \varepsilon}\right) h^{(1-\varepsilon) \beta} d x \leq C h^{\beta}
\end{aligned}
$$

since

$$
\int_{0}^{x_{1}} x^{-\varepsilon}\left(x_{1}-x\right)^{2 \varepsilon-1} d x=O\left(x_{1}^{\varepsilon}\right) .
$$

Altogether, we thus obtain

$$
\|w-\tilde{w}\|_{\tilde{H}^{1 / 2}} \leq\left\{\begin{array}{l}
C h \\
C h^{\beta / 2}
\end{array} \quad \text { for } \beta\left\{\begin{array}{l}
> \\
<
\end{array}\right\} \begin{array}{l}
2 \\
2
\end{array} .\right.
$$

From the Sobolev embedding theorem $H^{1 / 2}[0,1] \subset L^{p}[0,1]$ for all $p<\infty$ we obtain by duality $L^{q}[0,1] \subset \tilde{H}^{-1 / 2}[0,1]$ for all $q>1$, i.e.,

$$
\|u-\tilde{u}\|_{\tilde{H}^{-1 / 2}} \leq C_{q}\|u-\tilde{u}\|_{L^{q}} \text { for all } q>1 \text {. }
$$


For $1<q<2$ we observe

$$
\begin{aligned}
\mathscr{J}_{j}^{3} & :=\int_{x_{j}}^{x_{j+1}}\left|\frac{1}{x}(w-\tilde{w})(x)\right|^{q} d x \\
& \leq \begin{cases}C h^{\beta}(j+1)^{\beta-1} x_{j}^{-q} h^{\beta q / 2}(j+1)^{(\beta / 2-2) q} & \text { for } j \neq 0, \\
C\left(x_{1}^{1-q / 2}+x_{1} h^{-\beta q / 2}\right) & \text { for } j=0,\end{cases}
\end{aligned}
$$

and thus

$$
\mathscr{J}_{j}^{3} \leq C h^{\beta(1-q / 2)}(j+1)^{\beta(1-q / 2)-1-2 q} \text { for } j=0, \ldots, N-1 .
$$

This gives, for $1<q<2$,

$$
\|u-\tilde{u}\|_{L^{q}}^{q} \leq \sum_{j=0}^{N} \mathscr{J}_{j}^{3} \leq\left\{\begin{array}{l}
C h^{\beta(1-q / 2)} \\
C h^{2 q}
\end{array} \text { for } \beta\left\{\begin{array}{l}
< \\
>
\end{array}\right\} \begin{array}{l}
4 q /(2-q) \\
4 q /(2-q)
\end{array} .\right.
$$

Therefore, with an arbitrary $\varepsilon>0$,

$$
\|u-\tilde{u}\|_{\tilde{H}^{-1 / 2}} \leq\left\{\begin{array}{l}
C h^{2} \\
C h^{(\beta / 2)-\varepsilon}
\end{array} \quad \text { for } \beta\left\{\begin{array}{l}
> \\
<
\end{array}\right\} \begin{array}{l}
4 \\
4
\end{array} .\right.
$$

Now, from (2.10) and (2.12) the assertion of the lemma follows.

Combining Lemma 2.1 and Theorem 1.1 we obtain our final result on the theoretical convergence rate, Theorem 2.2 below. We assume that the grid $\tilde{\Delta}$ locally in a fixed one-sided neighborhood of each endpoint has the form (2.1) in a suitable coordinate representation.

THEOREM 2.2. There exist $N_{0} \in \mathbf{N}$ and $C \geq 0$ such that for all $g$ sufficiently smooth and $N \geq N_{0}$, the solutions $u \in H^{-1 / 2}(\Gamma)$ of $E q$. (1.1) and $u_{N} \in S_{\rho}^{1}(\tilde{\Delta})$ of (1.2) satisfy

$$
\left\|u-u_{N}\right\|_{\tilde{H}_{\rho}^{1 / 2}(\Gamma)} \leq C \begin{cases}h & \text { if } \beta>2, \\ h^{(\beta / 2)-\varepsilon} & \text { if } 1 \leq \beta<2 .\end{cases}
$$

For numerical experiments (see Section 3 ) it is more convenient to measure the error in a weighted $L^{2}$-norm. We have the following approximation property for our piecewise linear interpolant $\tilde{u}$ of $u$.

COROLlaRY 2.2. There exists a constant $C$ such that

$$
\|u-\tilde{u}\|_{L_{\rho}^{2}} \leq C \begin{cases}h^{\beta / 2} & \text { for } 1 \leq \beta<4 \\ h^{2} & \text { for } \beta>4\end{cases}
$$

where

$$
\|u\|_{L_{\rho}^{2}}:=\left\|\rho^{1 / 2} u\right\|_{L^{2}} .
$$

Proof. The estimate (2.13) follows with the arguments in the proof of Lemma 2.1 if one considers instead of $\mathscr{J}_{j}{ }^{3}$ in $(2.11)$ the following term:

$$
\mathscr{J}_{j}^{4}:=\int_{x_{j}}^{x_{j+1}} x\left|\frac{1}{x}(w-\tilde{w})(x)\right|^{2} d x \leq C \begin{cases}x_{1}+x_{1}^{2} h^{-\beta} \leq C h^{\beta} & \text { for } j=0, \\ h^{\beta}(j+1)^{\beta-5} & \text { for } j \neq 0 .\end{cases}
$$

Hence,

$$
\|u-\tilde{u}\|_{L_{\rho}^{2}}^{2} \leq \sum_{j=0}^{N} \mathscr{J}_{j}^{4} \leq C h^{\beta} \sum_{j=0}^{N}(j+1)^{\beta-5} \leq \begin{cases}C h^{\beta} & \text { for } \beta<4 \\ C h^{4} & \text { for } \beta>4\end{cases}
$$


3. Numerical Experiments. Below we present the numerical results of our collocation method (for the integral equation of the first kind with the single layer potential) for two examples: 1) lateral crack and 2) interior crack problems governed by the Laplacian. In both examples we construct the collocation solution using piecewise linear trial functions which are discontinuous at the corners. This method is implemented in [6] for plane domains with polygonal boundaries. The general program described in [6] applies directly to the situation in the examples below, i.e., even crack problems can be treated. As shown in Section 2, we need for our error analysis slightly different trial functions, namely the piecewise linear functions which are constant in the neighboring subintervals of the vertices. Nevertheless, numerical tests reveal for both kind of trial functions the same experimental order of convergence of the collocation solution. The theoretical orders follow immediately from (2.13).

(1) Lateral crack $\Gamma_{0}$ given by the straight line $0 \leq x \leq 1, y=0$ in a membrane $\Omega$ given by $-1 \leq x, y \leq 1$ : We convert the Dirichlet crack problem $(\tilde{\Gamma}$ denotes the boundary of the membrane)

$$
\begin{gathered}
\Delta v=0 \quad \text { in } \Omega, \\
v=\phi \quad \text { on } \Gamma=\Gamma_{0} \cup \tilde{\Gamma}, \quad \tilde{\Gamma}=\Gamma_{1} \cup \Gamma_{2} \cup \Gamma_{3} \cup \Gamma_{4} \cup \Gamma_{5}
\end{gathered}
$$

into the integral equation (1.1), i.e.,

$$
V u(z)=-\frac{1}{\pi} \int_{\Gamma} u(\varsigma) \log |z-\varsigma| d s(\varsigma)=g(z) \quad \text { on } \Gamma,
$$

where

$$
g(z)=\phi(z)-\frac{1}{\pi} \int_{\Gamma} \phi(\varsigma) \frac{\partial}{\partial n \zeta} \log |z-\varsigma| d s(\varsigma)
$$

and

$$
u=\left[\frac{\partial v}{\partial n}\right] \quad \text { on } \Gamma_{0} \quad \text { and } \quad u=\frac{\partial v}{\partial n} \quad \text { on } \tilde{\Gamma} .
$$

Here, $[\partial v / \partial n]$ denotes the jump of the normal derivative of $v$ across $\Gamma_{0}$.

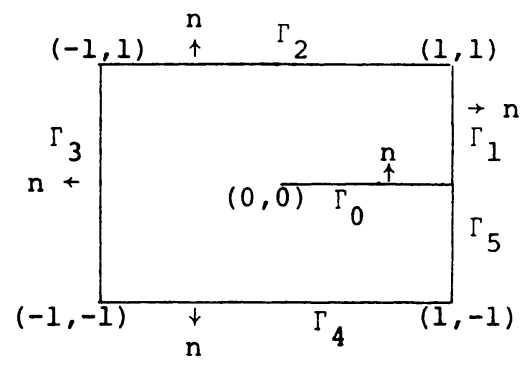

FIGURE 1

(For the direction of the normal vector $n$, see Figure 1. On $\tilde{\Gamma}, n$ is the outwardly directed normal, whereas on $\Gamma_{0}, n$ points in the positive $y$-direction.) For $v=\operatorname{Im} \sqrt{z}$ we compute approximations to the solution of (3.1) via the collocation scheme (1.3).

Table 1.1 contains the weighted $L^{2}$-error for Example 1 using piecewise linear trial functions which are discontinuous at the corners. We present in Table 1.2 the 
TABLE 1.1

Weighted $L^{2}$-error for Example 1 for piecewise linear trial functions.

\begin{tabular}{|c|c|c|c|c|}
\hline & \multicolumn{2}{|r|}{1} & \multicolumn{2}{|r|}{2} \\
\hline ISDES & $\begin{array}{l}\text { Weighted } \\
L^{2} \text {-Error }\end{array}$ & $\begin{array}{c}\text { Experimental } \\
\text { Convergence Rate }\end{array}$ & $\begin{array}{l}\text { Weighted } \\
L^{2} \text {-Error }\end{array}$ & $\begin{array}{c}\text { Experimental } \\
\text { Convergence Rate }\end{array}$ \\
\hline 4 & .2011 & .5132 & .1512 & 1.0155 \\
\hline 8 & .1409 & .5041 & .07479 & 1.0118 \\
\hline 16 & .09935 & .5013 & .03709 & 1.0035 \\
\hline 32 & .07019 & $\int$ & .01850 & J \\
\hline$\alpha$ * & & 0.5 & & 1.0 \\
\hline
\end{tabular}

$\alpha^{*}=$ theoretical order of convergence, $\beta=$ mesh grading parameter, NSDES = number of boundary elements per side.

TABLE 1.2

Weighted $L^{2}$-error for Example 1 for piecewise linear trial functions which are constant on subintervals next to corners/crack tips.

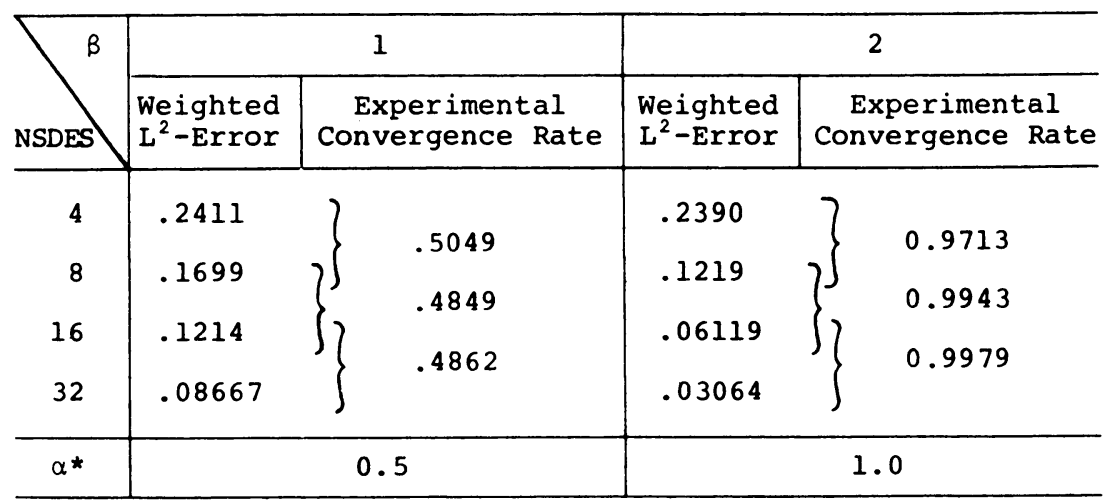

weighted $L^{2}$-error computed with piecewise linear trial functions which are constant on subintervals next to corners and crack tips.

(2) Interior crack $\Gamma_{0}$ given by $-1 \leq x \leq 1, y=0$ in an infinite membrane $\Omega=\mathbf{R}^{2} \backslash \bar{\Gamma}_{0}$. Here we consider the exterior Dirichlet problem

$$
\begin{gathered}
\Delta v=0 \quad \text { in } \mathbf{R}^{2} \backslash \bar{\Gamma}_{0} \\
v=\phi \quad \text { on slit } \Gamma_{0}=\{-1 \leq x \leq 1, y=0\}
\end{gathered}
$$

with

$$
\phi=\operatorname{Re}\left\{\sqrt{z^{2}-1}-z\right\}
$$


TABLE 1.3

Weighted $L^{2}$-error for Example 2 for piecewise linear trial functions.

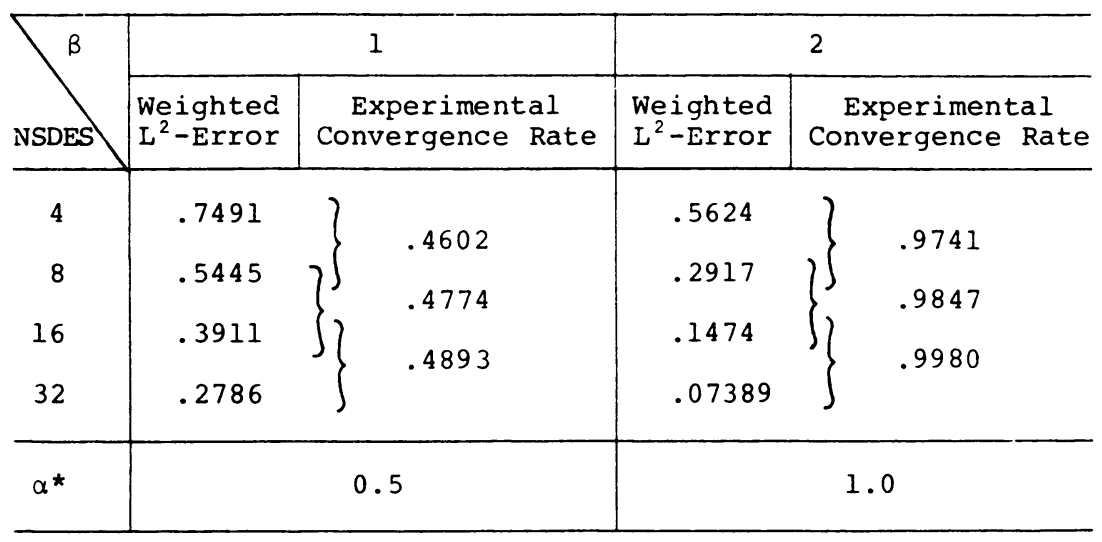

$\alpha^{*}=$ theoretical order of convergence, $\beta=$ mesh grading parameter, NSDES = number of boundary elements per side.

This boundary value problem is converted into the integral equation for $u=$ $[\partial v / \partial n]$, the jump of the normal derivative of $v$,

$$
\frac{1}{\pi} \int_{-1}^{1} u(z) \log |z-x| d x-\omega=2 z \quad \text { for } z \in \Gamma_{0} .
$$

Here we have used the condition $\int_{-1}^{1} u(x) d x=b$, where the constant $b$ is given by the asymptotic behavior of the solution $v$ at infinity,

$$
v=b \log |z|-\frac{1}{2} \omega+O(1 /|z|),
$$

and $\omega$ is a constant to be determined. In the above example, $v(z)=\sqrt{z^{2}-1}-z$ behaves like $O(1 /|z|)$ at infinity, yielding $b=\omega=0$. In Table 1.3 we list the weighted $L^{2}$-errors of the collocation solution for the above integral equation, where we set $b=0$ in the compatibility condition. For all cases we compute $\omega=10^{-12}$. We also include in Table 1.3 the results when using an exponentially graded mesh $\beta \neq 1$.

Fachbereich Mathematik

Technische Hochschule Darmstadt

D-6100 Darmstadt, West Germany

School of Mathematics

Georgia Institute of Technology

Atlanta, Georgia 30332

School of Mathematics

Georgia Institute of Technology

Atlanta, Georgia 30332

1. P.M. Anselone, Collectively Compact Operator Approximation Theory and Applications to Integral Equations, Prentice-Hall, Englewood Cliffs, N. J., 1971.

2. D. N. ARNOLD \& W. L. WENDLAND, "On the asymptotic convergence of collocation methods," Math. Comp., v. 41, 1984, pp. 349-381. 
3. D. N. ARNOLD \& W. L. WENDLAND, "The convergence of spline collocation for strongly elliptic equations on curves," Numer. Math., v. 47, 1985, pp. 317-341.

4. K. E. AtKInSON, A Survey of Numerical Methods for the Solution of Fredholm Integral Equations of the Second Kind, SIAM, Philadelphia, Pa., 1976.

5. C. T. H. BAKER, The Numerical Treatment of Integral Equations, Oxford University Press, Oxford, 1977.

6. M. Costabel, V. J. ERvin \& E. P. Stephan, "Experimental asymptotic convergence of collocation method for boundary integral equations on polygons," submitted to J. Comput. Mech.

7. M. Costabel \& E. P. STEPhaN, "Boundary integral equations for mixed boundary value problems in polygonal domains and Galerkin approximation," in Mathematical Models and Methods in Mechanics 1981, Banach Center Publications Vol. 15 (W. Fiszdon, K. Wilmański, eds.), PWNPolish Scientific Publishers, Warsaw, 1985, pp. 175-251. Short report in Advances in Computer Methods for Partial Differential Equations IV, IMACS, 1981, pp. 300-340.

8. M. COSTABEL \& E. P. STEPHAN, "On the convergence of collocation methods for boundary integral equations on polygons," Math. Comp., v. 49, 1987, pp. 461-478.

9. M. COSTABEL \& E. P. STEPHAN, "Collocation methods for integral equations on polygons," in Innovative Numerical Methods in Engineering (R. P. Shaw et al., eds.), Proc. 4th Internat. Sympos., Georgia Tech, Atlanta, GA, 1986, Springer-Verlag, Berlin and New York, 1986, pp. 43-50.

10. J. ElsChNeR, On Spline Collocation for Singular Integral Equations on an Interval, Seminar Analysis 1985/86, pp. 31-54, Karl-Weierstrass-Institut für Mathematik, Berlin, 1986.

11. I. C. Gohberg \& I. A. Feldman, Convolution Equations and Projection Methods for Their Solution, Transl. Math. Monos., vol. 41, Amer. Math. Soc., Providence, R. I., 1974.

12. R. HAGEN \& B. SilbermanN, "A finite element collocation method for bisingular integral equations," Applicable Anal., v. 19, 1985, pp. 117-135.

13. M. A. KRASnOsel'Skil Et Al., Approximate Solution of Operator Equations, Noordhoff, Groningen, 1972.

14. J. L. LiOns \& E. MAgenes, Non-Homogeneous Boundary Value Problems and Applications I, Springer, Berlin, 1972.

15. S. PRÖßDORF, "Ein Lokalisierungsprinzip in der Theorie der Spline-Approximation und einige Anwendungen," Math. Nachr., v. 119, 1984, pp. 239-255.

16. S. PRÖßDORF \& B. SILBERMANN, Gestörte Projektionsverfahren und einige ihrer Anwendungen, Abhandlungen der Akademie d. Wiss. der DDR, Abt. Mathematik, Nr. 6N, Akademie-Verlag, Berlin, 1978, pp. 229-237.

17. S. PRÖßDORF \& A. RATHSFELD, "A spline collocation method for singular integral equations with piecewise continuous coefficients," Integral Equations Operator Theory, v. 7, 1984, pp. $536-560$.

18. J. SARANEN \& W. L. WENDLAND, "On the asymptotic convergence of collocation methods with spline functions of even degree," Math. Comp., v. 45, 1985, pp. 91-108.

19. G. SCHMIDT, "On spline collocation for singular integral equations," Math. Nachr., v. 111, 1983, pp. 177-196.

20. W. L. WENDLAND, "Boundary element methods and their asymptotic convergence," in Theoretical Acoustics and Numerical Techniques (P. Filippi, ed.), CISM Lectures 277, Springer, Wien-New York, 1983, pp. 135-216. 